\title{
Price changing and inventory sharing in supply chain management
}

\author{
Kristina Šorić $^{1, \dagger}$, Višnja Vojvodić Rosenzweig ${ }^{1}$ and Jadranka \\ Kraljević ${ }^{2}$ \\ ${ }^{1}$ Zagreb School of Economics and Management, Jordanovac 110, 10000 Zagreb, Croatia \\ E-mail:〈ksoric@zsem.hr,vojvodic.visnja@gmail.com 〉 \\ ${ }^{2}$ Croatian Catholic University, Ilica 242, 10000 Zagreb, Croatia \\ E-mail: 〈jakicakr@gmail.com >
}

\begin{abstract}
The main task of supply chain management is to balance efficiency and effectiveness. Numerous operational management strategies are used to make a supply chain efficient, one such is inventory management. In this paper, we will consider a particular part of a supply chain consisting comprising a manufacturer and a retailer with the goal of minimizing associated inventory costs. We will focus on an inventory of final products determined as the difference between supply and demand and are expressed as a function of price, inflation rate and change in inflation rate resulting in the possible speculations. The manufacturer's inventory cost is a function of these same variables, with the retailer's inventory cost having the same function in addition to the margin. The problem is formulated as a dynamic game to share the speculation problem. The optimization problems to be solved are optimal control theory problems with objective functions in the form of integral functional with the integrand depending on the state function and its first and second derivative.
\end{abstract}

Keywords: supply chain, inventory management, risk sharing, optimal control theory

Received: October 17, 2016; accepted: March 19, 2017; available online: March 31, 2017

DOI: $10.17535 /$ crorr. 2017.0011

\section{Introduction}

In the last two decades, supply chain management has been a topic addressed in numerous studies and researches. Prior to the actual economic crises, one way to increase profit was to increase revenue. Nowadays, companies are looking for other ways of achieving profitability. Strategies such as minimizing cost, optimizing processes and establishing cooperation in a supply chain have been increasingly

\footnotetext{
$\dagger$ Corresponding author 
incorporated into business plans. In addition to these strategies, companies often speculate to increase the profit, and to avoid poor business results.

$\mathrm{E}$-commerce is a good example of price speculation, especially in regard to computer components and accessories, as well as fashion goods and toys. In these cases, we encounter speculation for reselling purposes. The second type of speculation is found in supply chains, including numerous entities and in opportunities to manipulate prices, costs, demand, information, inventory and deadlines. The distributors often retain stocks to create product scarcity and in turn stimulate demand and increase prices. The third type of speculation occurs in macroeconomic movements, without or with intervention from monetary authorities. Supply chain members may find themselves in a situation where loans are subject to uncertain interest rates and will incorporate this uncertainty in their prices. They may also expect discrete intervention from monetary authorities which in turn may impact prices. Moreover, we observe situations as prices decrease due to fall in consumption, something that creates a good environment for speculation.

The type of speculation addressed in this paper refers to supply chain management where demand and supply depend on product prices and prices trends. The main motive lies in the fact that supply chain members do not cooperate and mistrust each other. Even if theory suggests this behavior, cooperation is not common in practice. Moreover, speculation in supply chains may occur in the situations mentioned earlier.

Our contributions shall cover several points. We will look at a supply chain consisting of a manufacturer and a retailer. The manufacturer delivers products to the retailer. Some of the production remains in the manufacturer's inventory. The retailer purchases the products and upon receiving them immediately sells some of the products, with the remainder becoming part of the retailer's inventory. Hence, the manufacturer and retailer have their own inventories comprising the same product. Both want to minimize inventory costs and the shortage risks, i.e., maintain a high level of service and satisfying demand. Supply and demand depend on price and the price trends which in turn result in speculation on quantities, where inventory is defined as the difference between supply and demand. The problem is defined as the Stackelberg game, where the retailer is the leader and the manufacturer is the follower. The optimization problems to be solved are optimal control theory problems with objective functions in the form of integral functional and the integrands depending on the state function, as well as its first and second derivative.

In this paper, we will derive the necessary condition for the follower's optimal solution (i.e. the manufacturer), and derive the optimal price time path as well as discuss the conditions for this time path to have economic sense. The leader's optimal solution (i.e. the retailer) will be future work.

This paper is organized as follows. In Section 2 we give a brief review of the literature on speculations in supply chains. Section 3 develops the model in the form 
of a Stackelberg game. In Section 4 we derive and interpret the necessary and sufficient conditions for manufacturer's optimal price time path. In Section 5 we prove the positivity of the manufacturer's price time path, while Section 6 presents conclusions and future work.

\section{Literature review}

This section provides an overview of important literature in the area of speculations in supply chain management, especially when viewed as optimal control theory problems ${ }^{\ddagger}$ with the objective function in the form of a functional integral and the integrands depending on the state function, its first and second derivative. When considering this specific problem, we find that literature covering this issue is a bit scarce.

The literature we mostly relied on is paper [2] which considers the case of dual sales channels. Speculations in this case are possible because the price difference in two stages of a supply chain leads to the possibility of reselling. The authors examine the influence of speculations on a manufacturer's and a retailer's ordering decisions. In some cases, both the manufacturer and retailer benefit from speculations, whereas in some cases both are hurt by a high number of speculations. The problem is defined as a two-player game, the manufacturer and retailer maximizing their profits subject to stochastic primary demand.

Logistics problems involving service parts were considering in paper [5]. This kind of problem induces a strong interaction between network design and inventory sharing due to the high cost, low demand for parts and deadlines. The problem is defined as a non-linear integer programming problem which in fact presents a modified binary knapsack problem. The problem of inventory sharing is defined in general as two facilities in a supply chain where they endeavor to minimize facility, inventory and transportation costs in line with deadlines.

Inventory control subject to speculation was addressed in paper [3] where the authors studied the problem of determining inventory levels based on stochastic demand with the objective of minimizing the total cost as the sum of purchasing, holding and penalty costs for a single supply chain member. That is, they considered forward buying for a retailer opposing an expected increase in the supplier's price.

Paper [4] does not propose any mathematical model representing an optimization problem, but the authors did analyze the role of speculation in the respective price boom. Large amounts of speculative money were invested in different commodity derivatives resulting in significant and unwarranted upward pressure on commodity prices.

In [6] a model to allocate stock levels at warehouses, each facing Poisson demand is considered. The authors proposed a non-linear integer programming model and

\footnotetext{
\$ See $[1]$
} 
developed an enumerative method to solve it. The results show that the proposed inventory sharing strategy results in considerable cost reduction when compared to the no-sharing case and the method is quite efficient for the considered test problems.

As our summary above illustrates, and based on our knowledge, not many papers consider the inventory sharing problem in terms of optimal control theory. Moreover, from a mathematical point of view, only a few papers treat the optimal control theory problem using an objective function in the form of integral functional where the integrand depends on the state function, its first and second derivative.

\section{Modeling the problem}

As mentioned above, we have a supply chain consisting of a manufacturer and a retailer. The manufacturer produces products. Part of the products are delivered to the retailer while the remainder is located in the manufacturer's inventory. The retailer buys a certain number of the products. Some of it is sold immediately upon receiving the goods and the rest is located in the retailer's inventory.

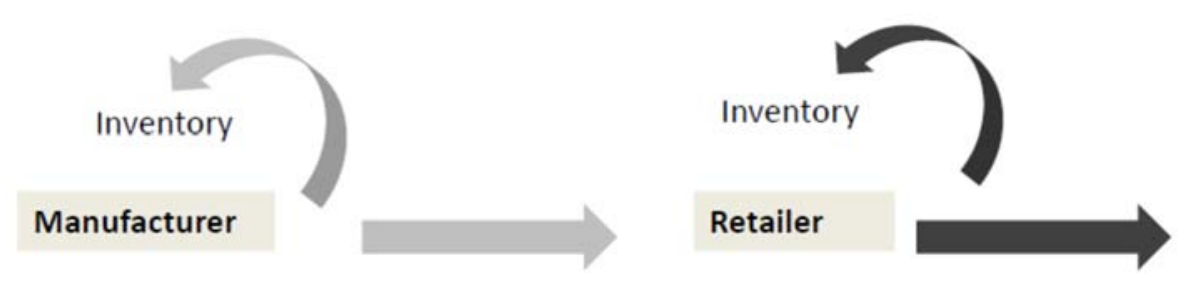

Figure 1: A supply chain consisting of a manufacturer and a retailer

The inventory is determined as the difference between supply and demand depending on the price and its trend represented by the price's first and second derivative. In general, supply and demand are given by

$$
\begin{aligned}
& Q_{s}=-\gamma+\delta p+u p^{\prime}+w p^{\prime \prime} \\
& Q_{d}=\alpha-\beta p+m p^{\prime}+n p^{\prime \prime},
\end{aligned}
$$

where $\alpha, \beta, \gamma, \delta>0$ and $m, n, u, w \in R$ are the speculative elements. The elements $m, n \in R$ are speculative in the that consumers decide whether to decrease demand as inflation increases or increase demand to create additional the inventory. The elements $u, w \in R$ are speculative in that the product supplier can increase supply and create inventory for reselling as inflation increases or the 
supplier can decrease supply as inflation increases. In line with this concept, the manufacturer's inventory at any given moment is

$$
Q_{s m}-Q_{d r}=-\gamma-\alpha+(\delta+\beta) p+(u-m) p^{\prime}+(w-n) p^{\prime \prime},
$$

where $p$ is the manufacturer's price, $Q_{s m}$ the manufacturer's supply and $Q_{d r}$ the retailer's demand. The retailer's inventory at any given moment is

$$
Q_{s r}-Q_{d c}=-\gamma-\alpha+(\delta+\beta)(1+i) p+(u-m)(1+i) p^{\prime}+(w-n)(1+i) p^{\prime \prime},
$$

where $i>0$ is the retailer's margin, $Q_{s r}$ the retailer's supply and $Q_{d c}$ the customers' demand.

We assume that the manufacturer and retailer divide only the speculative part of the inventory to optimally prevent price changes. Thus, let $k \in\langle 0,1\rangle$ be the manufacturer's share, and let $(1-k) \in\langle 0,1\rangle$ be the retailer's share in the speculative inventory.

The manufacturer's objective is to minimize its inventory cost over the period $[0, T]$ :

$$
\min _{p}^{T} \int_{0}^{T} c_{m} p(t)\left(-\gamma-\alpha+(\delta+\beta) p(t)+k(u-m) p^{\prime}(t)+k(w-n) p^{\prime \prime}(t)\right) d t^{\prime}
$$

where $c_{m} p(t)$ is the inventory cost as a percentage of its value.

The retailer's objective is to minimize its inventory over the period $[0, T]$ :

$$
\min _{k, i \geq i_{0}} \int_{0}^{T} c_{r}(1+i) p\left(-\gamma-\alpha+(\delta+\beta)(1+i) p(t)+(1-k)(u-m)(1+i) p^{\prime}(t)+(1-k)(w-n)(1+i) p^{\prime \prime}(t)\right) d t
$$

where $c_{r}(i+1) p(t)$ is the inventory cost as a percentage of its value and $i_{0}$ is some minimum margin for the retailer.

Since the manufacturer and retailer know that a certain amount of inventory has to exist in order to avoid certain risks in the supply chain, we determine a Stackelberg game ${ }^{\S}$ between manufacturer as the follower and the retailer as the leader as follows:

$\S$ See $[7]$ 


$$
\begin{gathered}
\min _{k, \geq_{0}}^{T} \int_{0} c_{r}\left((-\gamma-\alpha)(1+i) p(t)+(\delta+\beta)(1+i)^{2} p^{2}(t)+(1-k)(u-m)(1+i)^{2} p(t) p^{\prime}(t)+(1-k)(w-n)(1+i)^{2} p(t) p^{\prime}(t)\right) d t \\
\qquad i \in R, i \geq i_{0} \geq 0, k \in\langle 0,1\rangle \\
\min _{p} \int_{0}^{T} c_{m}\left((-\gamma-\alpha) p(t)+(\delta+\beta) p^{2}(t)+k(u-m) p(t) p^{\prime}(t)+k(w-n) p(t) p^{\prime \prime}(t)\right) d t
\end{gathered}
$$

\section{Solution to the manufacturer's problem}

To solve this optimization problem in the form of a Stackelberg game, we apply the concept of backwards induction. First, we solve the manufacturer's and then the retailer's problem.

We introduce the following notation: $A=\gamma+\alpha, B=\delta+\beta, C=k(u-m)$, and $D=k(w-n)$. The manufacturer's problem is now

$$
\min _{p} \int_{0}^{T} c_{m}\left(-A p+B p^{2}+C p p^{\prime}+D p p^{\prime \prime}\right) d t
$$

Some parts of the above integral are solved easily, i.e.

$$
\int_{0}^{T} C p p^{\prime} d t=C\left(\frac{1}{2}\left(p^{2}(T)-p^{2}(0)\right)\right)
$$

and

$$
\int_{0}^{T} D p p^{\prime \prime} d t=D\left(p(T) p^{\prime}(T)-p(0) p^{\prime}(0)\right)-D \int_{0}^{T} p^{\prime 2} d t
$$

If we introduce the notation

$$
K=C\left(\frac{1}{2}\left(p^{2}(T)-p^{2}(0)\right)\right)+D\left(p(T) p^{\prime}(T)-p(0) p^{\prime}(0)\right),
$$

the manufacturer's objective function is 


$$
c_{m} \int_{0}^{T}\left(-A p+B p^{2}+C p p^{\prime}+D p p^{\prime \prime}\right) d t=\int_{0}^{T}\left(-A p+B p^{2}-D p^{\prime 2}\right) d t+K
$$

where $p(T)$ and $p^{\prime}(T)$ are known as macroeconomic forecasts, and the corresponding problem becomes:

$$
\min _{p} \int_{0}^{T}\left(-A p+B p^{2}-D p^{\prime 2}\right) d t
$$

Using Euler-Lagrange, the necessary condition $\frac{d}{d t}\left(L-p^{\prime} \frac{\partial L}{\partial p^{\prime}}\right)=0$ for

$$
L=L\left(p, p^{\prime}\right)=-A p+B p^{2}-D p^{\prime 2},
$$

and assuming that $\frac{B}{D}<0$, we obtain the price time path

$$
p(t)=C_{1} e^{a t}+C_{2} e^{-a t}+\frac{A}{2 B} \text { where } a=\sqrt{-\frac{B}{D}} .
$$

\subsection{Interpretation of the existence conditions for manufacturer's price time path}

A sufficient condition for the minimum is

$$
\frac{\partial^{2} L}{\partial p^{\prime 2}}=-2 D>0 \Leftrightarrow D<0
$$

Thus, if $D<0$ then $\frac{B}{D}<0$. The assumption that $D<0$, where $D=k(w-n)$ means that $w-n<0$, i.e. the difference between the speculative elements in the manufacturer's supply function and the retailer's demand function is negative. Thus, a price change trend sensitivity for the manufacturer is less than the price change trend sensitivity for retailer, which is very reasonable since the retailer is more sensitive on price changes due to his flexibility. This condition is used to 
obtain the manufacturer's optimal price time path. If the condition is not satisfied for an optimal price time path, the manufacturer and retailer have to negotiate.

\section{Positivity of the manufacturer's price time path}

In Section 4, we obtained the manufacturer's price time path

$$
p(t)=C_{1} e^{a t}+C_{2} e^{-a t}+\frac{A}{2 B} .
$$

We will now prove its positivity. With $b=\frac{A}{2 B}, b>0, a>0$ and $t \in[0, T]$ we have

$$
\begin{gathered}
p(t)=C_{1} e^{a t}+C_{2} e^{-a t}+b \\
C_{1}=0.5\left(p(0)-b+\frac{1}{a} p^{\prime}(0)\right) \\
C_{2}=0.5\left(p(0)-b-\frac{1}{a} p^{\prime}(0)\right) .
\end{gathered}
$$

Further

$$
\begin{gathered}
p^{\prime}(t)=a\left(C_{1} e^{a t}-C_{2} e^{-a t}\right) \\
p^{\prime \prime}(t)=a^{2}\left(C_{1} e^{a t}+C_{2} e^{-a t}\right) .
\end{gathered}
$$

Also $p^{\prime}(t)=0$ for $e^{a t}=\frac{C_{2}}{C_{1}} e^{-a t}$, i.e.

$$
e^{2 a t}=\frac{C_{2}}{C_{1}}
$$

The time path $p=p(t)$ has a stationary point $t^{*}$ if constants $C_{1}$ and $C_{2}$ have the same sign. Thus

$$
2 a t^{*}=\ln \frac{C_{2}}{C_{1}}
$$

The stationary point is negative if $\frac{C_{2}}{C_{1}}<1$ and positive if $\frac{C_{2}}{C_{1}}>1$.

The time path $p=p(t)$ has an inflection point $\tilde{t}$ if constants $C_{1}$ and $C_{2}$ have different signs. Thus $2 a \tilde{t}=\ln \frac{-C_{2}}{C_{1}}$. The inflection point is negative if $\frac{-C_{2}}{C_{1}}<1$ and positive if $\frac{-C_{2}}{C_{1}}>1$. 


\subsection{Case $\mathrm{t}=0, p^{\prime}(0)>0$.}

From $p^{\prime}(0)>0$ it follows that

$$
C_{1}>C_{2}
$$

1a) If $C_{1}, C_{2}>0$ we have $p(t)>0$ and $p(t)$ is a convex function as a positive combination of two convex functions. It has a global minimum in its stationary point. On the other hand, due to (5) and the assumption that the constants are positive, we have $1>\frac{C_{2}}{C_{1}}>0$. It follows that $\ln \frac{C_{2}}{C_{1}}<0$. The stationary point is negative. Hence, the time path $p(t)$ is an increasing function and

$$
\begin{aligned}
& \max _{0 \leq t \leq T} p(t)=p(T) \\
& \min _{0 \leq t \leq T} p(t)=p(0) .
\end{aligned}
$$

1b) If $C_{1}, C_{2}<0$ then $p(t)$ is a concave function and reaches a maximum at its stationary point. Due to (5) and the assumption that the constants are negative, we have $\frac{C_{2}}{C_{1}}>1$. It follows that $\ln \frac{C_{2}}{C_{1}}>0$, or the stationary point in which the maximum is obtained is positive. Thus, the stationary point is $t^{*}=\frac{1}{2 a} \ln \frac{C_{2}}{C_{1}}$. Also, $p\left(t^{*}\right)>p(0)>0$.

1b1) If $t^{*}>T$, the time path $p(t)$ is an increasing function on $[0, T]$ and

$$
\begin{aligned}
& \max _{0 \leq t \leq T} p(t)=p(T) \\
& \min _{0 \leq t \leq T} p(t)=p(0) .
\end{aligned}
$$

It follows that $p(t)>0$.

1b2) If $0<t^{*}<T$ we have

$$
\begin{aligned}
& p(0)<p(t)<p\left(t^{*}\right) \text { for } 0<t<t^{*} \\
& p\left(t^{*}\right)>p(t)>p(T) \text { for } t^{*}<t<T .
\end{aligned}
$$

1c) If the constants $C_{1}$ and $C_{2}$ have different signs, on account of (5) we have $C_{1}>0, C_{2}<0$ and $p(t)$ is an increasing function on $[0, T]$,

$$
\begin{aligned}
& \max _{0 \leq t \leq T} p(t)=p(T) \\
& \min _{0 \leq t \leq T} p(t)=p(0) .
\end{aligned}
$$


It follows that $p(t)>0$.

\subsection{Case $\mathrm{t}=0, \boldsymbol{p}^{\prime}(0)<0$.}

From $p^{\prime}(0)<0$ it follows that

$$
C_{1}<C_{2}
$$

2a) If $C_{1}, C_{2}>0$, it follows that $p(t)>0$ and $p(t)$ is a convex function as a positive combination of two functions. It obtains the global minimum at the stationary point. On the other hand, due to (6) and the assumption that the constants are positive, we have

$$
\frac{C_{2}}{C_{1}}>1
$$

and $\ln \frac{C_{2}}{C_{1}}>0$. The stationary point is positive. If the global minimum is in the interval $[0, T]$ then the function decreases till $t^{*}$ and subsequently increases. If $t^{*}>T$ the function decreases from $p(0)$ till $p(T)$.

2b) If $C_{1}, C_{2}<0$, then $p(t)$ is a concave function and has a global maximum at its stationary point it. On the other hand, due to (6) and the assumption that the constants are negative, we have $1>\frac{C_{2}}{C_{1}}>0$ and thus $\ln \frac{C_{2}}{C_{1}}<0$. The stationary point is negative. The time path $p(t)$ decreases in the interval $[0, T]$. It follows

$$
\min _{0 \leq t \leq T} p(t)=p(T)>0 .
$$

Thus $p(t)>0$.

2c) If constants $C_{1}, C_{2}$ have different signs due to (6), we have $C_{1}<0, C_{2}>0$ and $p(t)$ decreases on $[0, T]$. It follows that

$$
\min _{0 \leq t \leq T} p(t)=p(T)>0
$$

Thus $p(t)>0$.

\subsection{Case $\mathrm{t}=0, p^{\prime}(0)=0$.}

Now

$$
\begin{gathered}
C_{1}=C_{2}=\hat{C} . \\
p(t)=\hat{C}\left(e^{a t}+e^{-a t}\right)+b
\end{gathered}
$$




$$
\hat{C}=0.5(p(0)-b)
$$

3a) If $\hat{C}>0$ i.e. $p(0)>b$, we have $p(t)>0$ and $p^{\prime}(t)=a \hat{C}\left(e^{a t}-e^{-a t}\right)>0$ for $\mathrm{t}>0$. Thus $p(t)$ is an increasing and convex function on $[0, T]$ and increases from $p(0)$ to $p(T)$.

3b) If $\hat{C}<0$, i.e. $p(0)<b$, we have $p^{\prime}(t)=a \hat{C}\left(e^{a t}-e^{-a t}\right)<0$ for $\mathrm{t}>0$.

Thus $p(t)$ is a decreasing and concave function on $[0, T]$. It decreases from $p(0)$ to $p(T)>0$ and

$$
\min _{0 \leq t \leq T} p(t)=p(T)>0
$$

Thus $p(t)>0$.

3c) If $\hat{C}=0$ i.e. $p(0)=b$ we have $p(t)=b>0$ for $t \in[0, T]$.

Remark. For the considered interval $[0, T]$ we assume that $p(T)>0$ due to the assumption of a macroeconomic forecast.

\section{Conclusions and future work}

In this paper, we have considered a particular supply chain consisting of a manufacturer and a retailer, both having the goal of minimizing their inventory costs. Inventory is determined as the difference between supply and demand, which are functions of price, inflation rate and inflation rate changes and resulting in possible speculation. To mitigate the risk, these two players can share the speculative part of the inventory. The problem is modeled as a Stackelberg game where the manufacturer is the follower and the retailer is the leader.

We derived the price time path by minimizing the manufacturer's inventory cost under certain conditions. These conditions require that the retailer is more sensitive to price trends than the manufacturer, which we find to be reasonable. Finally, we proved that the price time path is positive, assuming that a given and positive forecasted price at the end of the considered period.

In future research, the optimal margin time path for the retailer and the optimal share of inventory for both players should be derived. In addition, the problem can also incorporate reversed roles, where the manufacturer is the leader and the retailer is the follower.

The problem of inventory sharing can also be viewed also as a multicriteria optimization problem and the results compared to the results from the problem modeled as a Stackelberg game. 


\section{References}

[1] Clarke, F. (2013). Functional analysis, calculus of variations and optimal control. Graduate Texts in Mathematics, Springer-Verlag, London

[2] Feng, T. and Geunes, J. (2014). Speculation in a two-stage retail supply chain. IIE Transactions, 46:12, 1315-1328, DOI: 10.1080/0740817X.2014.904975

[3] Gavirneni, S. and Morton, T.E. (1999). Inventory control under speculation: myopic heuristics and exact procedures. European Journal of Operational Research, 117, 211-221

[4] Irwin, S.H., Sanders, D.R. and Merrin, R.P. (2009) Devil or angel? The role of speculation in the recent commodity price boom (and bust). Journal of Agricultural and Applied Economics, 41 (2), 377-391

[5] Iyoob, I.M. and Kutanoglu, E. (2013). Inventory sharing in integrated network design and inventory optimization with low-demand parts. European Journal of Operational Research, 224, 497-506

[6] Mahajan, M and Kutanoglu, E. (2009). An inventory sharing and allocation method for a multi-location service parts logistics network with time-based service levels. European Journal of Operational Research, 194, 728-742

[7] Osborne, M. J. (2004). An introduction to game theory, Oxford University 